\title{
Epithelial-mesenchymal transition and drug resistance in breast cancer (Review)
}

\author{
JING HUANG, HONGZHONG LI and GUOSHENG REN \\ Chongqing Key Laboratory of Molecular Oncology and Epigenetics, The First Affiliated Hospital \\ of Chongqing Medical University, Chongqing 400010, P.R. China
}

Received April 30, 2015; Accepted June 2, 2015

DOI: 10.3892/ijo.2015.3084

\begin{abstract}
Breast cancer is the leading cause of cancer death in women worldwide. Insensitivity of tumor cells to drug therapies is an essential reason arousing such high mortality. Epithelial-mesenchymal transition (EMT) is defined by the loss of epithelial characteristics and the acquisition of a mesenchymal phenotype. It is well known that EMT plays an important role in breast cancer progression. Recently, mounting evidence has demonstrated involvement of EMT in antagonizing chemotherapy in breast cancer. Here, we discuss the biological significance and clinical implications of these findings, with an emphasis on novel approaches that effectively target EMT to increase the efficacy of anticancer therapies.
\end{abstract}

\section{Contents}

1. Introduction

2. Drug resistance in breast cancer

3. The potential mechanism of EMT

4. EMT transcriptional factors and drug resistance in breast cancer

5. EMT-related cytokines and drug resistance in breast cancer

6. EMT-related signal pathways and drug resistance in breast cancer

7. Certain genes involved in EMT and drug resistance in breast cancer

8. EMT, CSCs and drug resistance in breast cancer

9. EMT, microRNAs and drug resistance in breast cancer

10. Clinical prognostic of EMT and potential EMT-targeted therapy for breast cancer

11. Concluding remarks and future perspectives

Correspondence to: Professor Guosheng Ren, Chongqing Key Laboratory of Molecular Oncology and Epigenetics, The First Affiliated Hospital of Chongqing Medical University, 1 Youyi Road, Yuzhong, Chongqing 400010, P.R. China

E-mail: rengs726@126.com

Key words: breast cancer, epithelial-mesenchymal transition, drug resistance, cancer stem cells, microRNA

\section{Introduction}

Breast cancer is the most frequently diagnosed cancer and the leading cause of cancer death in women worldwide, accounting for $\sim 23 \%$ of the total new cancer cases and $14 \%$ of the total cancer deaths (1). It is well known that breast cancer is a heterogeneous disease that can be classified by microscopic appearance and molecular profiles such as estrogen receptor (ER), progesterone receptor (PR) and human epidermal growth factor receptor 2 (HER2) which correlate with diverse clinical outcomes and responses to treatment. Systemic treatment for breast cancer, including conventional cytotoxic therapy (paclitaxe, doxorubicine, cyclophosphamide, fluorouracil, cis-platinum), endocrine treatment (tamoxifen, fulvestrant, letrozole, anastrozole), and targeted agents such as trastuzumab, plays an essential role in reducing mortality rate and prolonging survival time in patients with breast cancer $(2,3)$. However, resistance to therapeutic agents remains a consistent obstacle in terms of treatment success, while the underlying mechanism of drug resistance remains enigmatic $(4,5)$.

Epithelial-mesenchymal transition (EMT) is a biologic process by which epithelial cells lose their cell polarity and cell-cell adhesion, and gain migratory and invasive properties to become mesenchymal cells. A growing body of literature supports that EMT is closely linked to the progression of breast cancer, which includes enhanced migratory and invasive capacity, and elevated stemness of cancer cells $(6,7)$. Now, emerging evidence suggests that EMT is also involved in treatment resistance in breast cancer $(8,9)$. This review presents the events that involve the impact of EMT on drug resistance in breast cancer, helping understand the generation of treatment resistance and seek potential approaches to reverse the process.

\section{Drug resistance in breast cancer}

In breast cancer treatment, conventional cytotoxic agents used alone or in combination weaken and destroy cancer cells in the body. However, resistance to chemotherapy is a major hurdle in the management of breast cancer. Some patients exhibit intrinsic resistance to chemotherapy, while other patients, although initially sensitive to chemotherapy, eventually develop acquired resistance, even after combination therapy. At present, it is well accepted that the mechanisms of chemoresistance may mainly contain decreasing uptake of 
water-soluble drugs, various changes in cells that affect the capacity of cytotoxic drugs to kill cells and increasing energydependent efflux of hydrophobic drugs that can easily enter the cells by diffusion through the plasma membrane (10). Moreover, topoisomerase poisons, altered expression of drugmetabolizing enzymes and drug-conjugate export pumps, suppression of apoptotic pathways and host-tumor-drug interactions also contribute to chemoresistance (11). Among these, the most significant is the increased efflux of hydrophobic drugs which are regulated by a family of energy-dependent transporters, known as ATP-binding cassette (ABC) transporters including P-glycoprotein (P-gp, also known as ABCB1 or MDR1), multidrug resistance protein (MRP) 1-7, lung resistance-related protein (LRP) and breast cancer resistance protein (BCRP) (12).

Tamoxifen (TAM) is the usual endocrine (anti-estrogen) therapy inducing objective response or disease stabilization in breast cancer patients with $\mathrm{ER}^{+}$tumors. The pharmacologic action of tamoxifen is that it binds to the estrogen receptor and induces dimerization and DNA binding to finally inactivate it (13). Nevertheless, about half of $\mathrm{ER}^{+}$patients with advanced disease and nearly all patients with metastatic disease fail to respond to first-line TAM treatment. Approximately $40 \%$ of patients receiving TAM as adjuvant therapy experience tumor relapse and die from their disease, and a third of women treated with TAM for 5 years develop recurrent disease within 15 years (14). TAM resistance might arise as a consequence of loss of expression or function of $\mathrm{ER} \alpha$, including autophosphorylation, modulation by activation of transmembrane tyrosine kinase receptors and interaction between downstream signal transduction pathways (15).

Trastuzumab (herceptin), a humanized, recombinant monoclonal antibody that selectively binds with high affinity to the extracellular domain of HER2, has been proved to exert antitumor effects in cancer models and patients with HER2-amplified breast cancer. The addition of trastuzumab to adjuvant chemotherapy can impressively reduce the recurrence rate (16). However, some patients with HER2overexpressing breast cancer do not respond to trastuzumab therapy. There is only $26 \%$ response rate in women diagnosed with HER2-positive metastatic breast cancer and treated with trastuzumab as a single first-line agent. That is, $>70 \%$ of HER2-overexpressing metastatic breast carcinomas display a resistance to trastuzumab (17). The mechanisms underlying the resistance phenotype are not well understood. Increased production of insulin-like growth factor, dysregulation of $\mathrm{p} 27$, overexpression of epidermal growth factor receptor (EGFR) with activation of the Akt pathway and decreased PTEN function may contribute to this process (18).

\section{The potential mechanism of EMT}

EMT refers to a complex molecular and cellular program by which epithelial cells shed their differentiated characteristics, including cell-cell adhesion, planar/apical-basal polarity, and lack of motility, and instead acquire mesenchymal features. It has been described as the transition taking place in epithelial cancer cells, which may lead to cancer invasion, resistance to anoikis and systemic cancer cell dissemination (19). During the acquisition of EMT characteristics, cells undergo actin cyto- skeleton reorganization, decrease in the expression of proteins that promote cell-cell contact such as E-cadherin and occludin, and gain in the expression of mesenchymal markers such as vimentin, fibronectin and $\mathrm{N}$-cadherin, as well as increased activity of matrix metalloproteinases (MMPs) like MMP-2, MMP-3 and MMP-9, which are associated with an invasive phenotype (20). The tumor microenvironment comprised of extracellular matrix, cells, and soluble factors plays a critical role in EMT induction and further in tumor metastasis $(21,22)$. Several kinds of stromal cell subtypes, such as macrophages and fibroblasts, contribute to tumor progression through induction of EMT $(23,24)$. EMT can also be triggered by adverse conditions such as hypoxia and a diverse set of extracellular stimuli including tumor necrosis factor $\alpha$ (TNF- $\alpha$ ), transforming growth factor $\beta$ (TGF- $\beta$ ), epithelial grow th factor family member (EGF), fibroblast growth factor (FGF), insulin growth factor (IGF), platelet derived growth factor (PDGF), and components of the extracellular matrix such as collagen and hyaluronic acid (21). Signal transduction pathways including Wnt, Notch, nuclear factor-kappa $\mathrm{B}(\mathrm{NF}-\kappa \mathrm{B})$, mitogen-activated protein kinase (MAPK) and phosphatidyl inositol 3-kinase (PI3K) pathways can coordinate EMT programs. Different stimuli induce a multitude of signal pathways that converge on several EMT-inducing transcriptional factors including Snail, Slug, Twist, Zeb1, Zeb2. All of the factors are capable of repressing E-cadherin directly or indirectly when overexpressed in cultured epithelial cells (25) (Fig. 1).

\section{EMT transcriptional factors and drug resistance in breast cancer}

It is well established that Snail family proteins (Snail and Slug) are the key regulatory elements of EMT along with the control of expression of many genes. Snail is involved in the EMT that not only takes place concomitantly with the acquisition of invasive properties in tumors, but also has been related to other cancer hallmarks such as the gain of unlimited replication potential, a greater resistance to apoptosis and even the evasion of immunosurveillance (26). Vega et al found that Snail attenuated the cell cycle and conferred resistance to cell death induced by the withdrawal of survival factors and proapoptotic signals (27). In another study, aberrant expression of Snail or Slug in breast adenocarcinoma cells was observed to protect against apoptosis induced by genotoxic stress, which might be associated with direct transcriptional repression of genes taking part in many aspects of programmed cell death (28). Chen et al revealed that MCF-7 breast cancer cells transfected with eukaryotic expression vector pCDNA3.1-Snail showed EMT with BCRP-mediated multidrug resistance (29). Similarly, it was reported that overexpression of Snail accelerated adriamycin induction of multidrug resistance through increasing the expression of P-gp (30). In paclitaxel, docetaxel, or doxorubicin resistant MCF-7 cell lines, Slug expression was upregulated and ER was downregulated, resulting in the repression of E-cadherin and occludin, and elevation of $\mathrm{N}$-cadherin and vimentin $(31,32)$. MCF-7 cells with ER deprivation were unresponsive to addition of estradiol and TAM and acquired the EMT state (33). It was shown that decrease in the estrogen dependency of breast cancer cells was accompanied by an increased expression and activity 

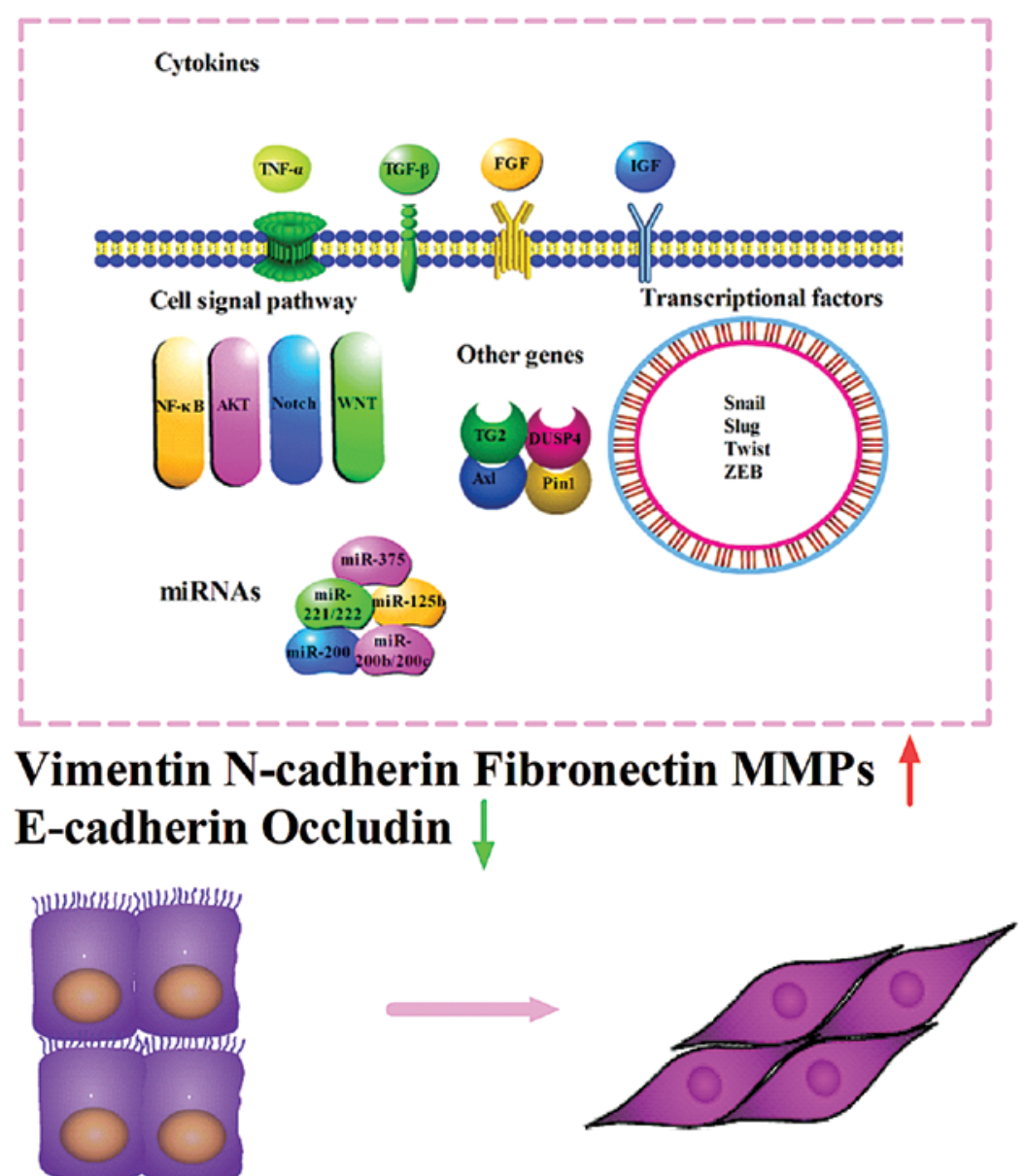

More invasive phenotype

Resistance to apoptosis

Elevated expression of $\mathrm{ABCs}$

Altered ER expression or EGFR/HER2

\section{Drug resistance}

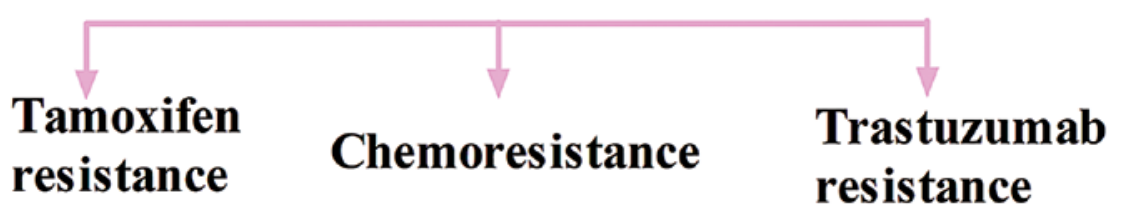

Figure 1. Brief representation of the role of transcriptional factors, cytokines, cell signal pathways, miRNAs and some other genes in EMT and drug resistance in breast cancer.

of Snail, and demonstrated the involvement of Snail in the negative regulation of ER (34). Further findings demonstrated that Snail could repress ER- $\alpha$ expression by direct interaction with regulatory DNA sequences at the ESR1 locus in breast cancer cell lines (35). In addition, many ER $\alpha$-negative lines which were also E-cadherin-negative (e.g., MDA-MB-468 and MDA-MB-231) exhibited high Slug expression. It is indicated that ligand-activated $\mathrm{ER} \alpha$ formed a transcriptional inhibitory complex comprised of nuclear receptor co-repressor $(\mathrm{N}-\mathrm{CoR})$ and histone deacetylase 1 (HDAC1) which bonds to the Slug promoter and directly suppresses Slug, which is one of the crit- ical members in Slug-E-cadherin-EMT pathway (36). Highly invasive breast cancer cell lines expressed elevated levels of Twist, which upregulated the transcription of Akt-2 to promote cell survival and resistance to paclitaxel (37). Li et al proved that adriamycin induced EMT and apoptosis in MCF-7 cells, while only cells undergoing EMT displayed multidrug resistance. Twist1 suppression prevented the drug-induced P-gp expression, concomitant with partial reduction in resistance to paclitaxel, vincristine and bleomycin (38). It seems that EMT induction simultaneously upregulates the expression of several $\mathrm{ABC}$ transporters, which lead to mutidrug resistance in human 
breast cancer cells. There were binding sites for several EMT transcription factors (Snail, Slug, Twist and FOXC2) in 16 ABC transporters, while CHIP analysis further revealed that Twist directly bound to E-boxes in the promoter region of ABCC4 and ABCC5 in MCF-7 cells transfected with Twist (39).

\section{EMT-related cytokines and drug resistance in breast cancer}

TGF- $\beta$ is one of the most potent and better-studied inducers of EMT, acts through serine-threonine kinase receptors to phosphorylate the cytoplasmic Smads which activates E-cadherin repressors of the Snail family. A recent study indicated that adverse activation of TGF- $\beta$ pathway by chemotherapeutics in the breast cancer cells or elevated TGF- $\beta$ levels in tumor microenvironment might lead to EMT and generation of cancer stem cells, resulting in the resistance to chemotherapy (40). The evidence found cis-platinum treatment of MDA-MB-231 breast cancer cells increased both TGF- $\beta$ mRNA levels and the secretion of active TGF- $\beta$, which enhanced growth arrest that facilitated repair of damage, thus rendering these cells resistant to cis-platinum killing (41). In the report of López-Díaz et al, TGF- $\beta$ was shown protected cells from DoxR, 5 -fluorouracil and paclitaxel-induced cell death specifically though Smad 4-mediated complex (42). Moreover, TGF- $\beta$ pretreatment was able to attenuate the TAM cytotoxic effect and decrease the apoptosis ratio in breast cancer (43). It was reported that TGF- $\beta$ increased ErbB/PI3K activation in BT474 and SKBR3 cells, and desensitized the cells to trastuzumab-mediated growth inhibition (44). TNF- $\alpha$ is another inflammatory cytokine linked to both EMT and drug resistance. MCF-7TN-R cells which were generated by prolonged and progressive exposure of MCF-7 cells to TNF- $\alpha$ underwent progressive EMT changes, and represented a model of transition to a multidrug resistant and increased tumorigenic phenotype. In addition, some growth factors which induce EMT may also take part in acquired resistance by various patterns. IGF-1 was proposed to transmit signals via both the PI3K and MAPK pathways, then resulted in the extracellular activation of MMPs which were capable of promoting latent TGF- $\beta 1$-induced EMT, further rescued breast cancer cells from chemotherapy-induced cell death $(45,46)$. It was also demonstrated that IGF-1 stimulated phosphorylation of HER-2 exclusively in the trastuzumab resistant cells. Antibody-mediated blockade of insulin growth factor repector (IGF-1R) disrupts IGF-1R interaction with HER-2 and restores trastuzumab sensitivity (47). FGF expression was found as a stronger predictor of paclitaxel resistance, compared to P-gp, p53, or Bcl-2 in patients with breast cancer (48). With the interaction with ER-activated pathways, FGF receptor-mediated signaling drives autonomous growth which would be refractory to TAM therapy (49). Regarding the induction of FGF on EMT, these results suggested that EMT might be involved in the FGF-mediated chemoresistance process.

\section{EMT-related signal pathways and drug resistance in breast cancer}

Many signaling pathways which have significant regulating effects on EMT are closely involved in drug resistance. Genomic Region Enrichment was performed to find increased secretase activity which may account for an increased Notch signaling in endocrine resistant breast cancer cells (50). PF-03084014 which inhibits Notch signaling by reducing Notch intracellular domain (NICD) and Notch target genes Hes-1 and c-Myc in both cells and tumors prominently enhanced the antitumor activity of docetaxel in MDA-MB-231 xenograft model through suppressing expression of survivin and myeloid cell leukemia sequence 1 (MCL1), reducing ABCB1 and ABCC2, upregulating BIM and reversing the EMT phenotype (51). Notch may be an important target in trastuzumab-resistant, HER-2 ${ }^{+}$breast cancer. Growth of trastuzumab-resistant cells was completely inhibited by combining trastuzumab plus Notch-1 siRNA (52). The NF- $\kappa$ B pathway is emerging as an essential regulator of EMT in cancer cell lines acting through the induction of Snail transcription and protein stabilization. Constitutively active $\mathrm{NF}-\kappa \mathrm{B}$ was also discovered to play a key role in resistance to death-inducing stimuli, including chemotherapeutic agents (53). NF- $\kappa \mathrm{B}$ inhibitors were found to sensitize breast cancer cells to doxorubicin (54). Previous studies also showed that phosphorylation and overexpression of NF- $\kappa \mathrm{B}$ caused an increase in ER-mediated transcription associated with endocrine resistance. As a positive regulator of Snail in breast cancer cells, simultaneous inhibition of $\mathrm{NF}-\kappa \mathrm{B}$ by RNA interference resulted in marked increase of cell response to antiestrogen TAM (55). Furthermore, the activation of MAPK and PI3K pathways was also involved in the adaptation of ER-positive breast cancer cells to estrogen deprivation by contributing to ER hypersensitivity and were associated with endocrine resistance (56). High PI3K/Akt activity has been associated with resistance to trastuzumab in HER2-overexpressing cells and primary tumors (57). Additionally, it was revealed that a number of canonical and non-canonical Wnt genes (DKK1, JUN, PORCN, CSNK1A1 and MYC) were significantly increased in the TAM-resistant cells. The Wnt inhibitor, IWP-2, resulted in decreased expression of vimentin and Twist (58). Wnt3 acting as a key mediator in the localization of $\beta$-catenin controlled EMT-like transition and activation of EGFR in trastuzumab resistant cells (59).

\section{Certain genes involved in EMT and drug resistance in breast cancer}

Transglutaminase 2 (TG2), a pro-inflammatory protein implicated in diverse physiological and pathological processes, was reported to induce EMT in MCF-10A cells and confer resistance to doxorubicin as an important downstream mediator of TGF- $\beta$ (60). Dual specificity phosphatase 4 (DUSP4) is a member of the dual specificity phosphatase family, which inactivates target kinases through dephosphorylating phosphoserine/threonine and phosphor tyrosine residues. Liu et al discovered that knockdown of DUSP4 increased the chemosensitivity of MCF-7 and MCF-7/ADR breast cancer cells to doxorubicin, and MCF-7/ADR cells with high levels of DUSP4 had a mesenchymal phenotype (61). Pin1, a peptidyl-prolyl isomerase, was overexpressed in TAM-resistant (TAMR) MCF-7 cells. Pin1 siRNA treatment resulted in decreased Snail transcription and the expression of EMT markers. It was inferred that Pin1 might take part in EMT by affecting PTEN expression and the subsequent PI3-kinase-Akt-dependent GSK-3 $\beta$ inactivation (62). Axl is a transmembrane tyrosine 
kinase receptor, activated by either its ligand-growth arrest specific 6 or extracellular domain-mediated dimerization or cross-talk with human EGFR2. It was shown that Axl induced EMT as a upstream factor in normal and immortalized human mammary epithelial cells in an apparent positive feedback loop mechanism and regulate breast cancer stem cells (BCSCs) self-renewal and chemoresistance (63).

\section{EMT, CSCs and drug resistance in breast cancer}

Cancer stem cells (CSCs), or tumor-initiating cells have been identified as having the ability to form mammosphere, self-renew, exhibiting the $\mathrm{CD} 44^{+} / \mathrm{CD} 24^{-}$or high aldehyde dehydrogenase $\left(\mathrm{ALDH}^{+}\right)$cell surface maker profile and being associated with invasion, relapse and drug-resistance (64). The stem cells refractory to therapies is mainly because CSCs are known to express increased levels of related members of ABC transporter family and anti-apoptotic proteins (65). Furthermore, these cells are hypothesized to be largely quiescent and slow cycling, which help escape from typical cytotoxic agents (66). BCSCs with high expression of ALDH can also help metabolize cytotoxic drugs (67). Morel et al were the first to present evidence linking EMT to BCSCs. It was shown that induction of EMT in transformed mammary epithelial cells generated cells with BCSCs properties (68). This was also corroborated in epithelial breast cancer cells of mouse models $(69,70)$. Circulating tumor cells from metastatic breast cancer patients have shown EMT and tumor stem cell characteristics (71). Basal-like breast cancers, which are enriched for $\mathrm{CD}_{4} 4^{+} / \mathrm{CD} 24^{-}$cells, are found to exhibit EMT features that might account for their aggressive clinical behavior and metastatic propensities. Moreover, a new subtype called

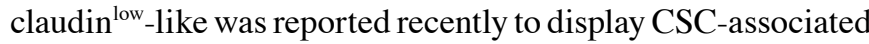
features. In addition, metaplastic tumors which are highly chemoresistant and aggressive are indicated to share molecular similarities with CSCs (72). Both the metaplastic and claudinlow-like tumors are closely related to the EMT core signatures (73). These results support a close connection between EMT and gain of CSC-like properties. Besides, stem-like cells can be generated from differentiated transformed mammary epithelial cells via EMT in vitro, suggesting that EMT plays an active role in generating CSCs in human breast tumors. HMLE cells acquired the CD $44^{\text {high }} / \mathrm{CD} 24^{\text {low }}$ stem cell profile, after stimulated by TGF- $\beta$ or in response to constitutive expression of either Twist or Snail (74). The loss of E-cadherin expression that transpires during EMT reinforces these events by permitting the nuclear translocation of $\beta$-catenin and its stimulation of CD44 expression (75). Overexpression of Twist in breast cancer cells was demonstrated to promote the generation of a breast cancer stem cell phenotype characterized by the high expression of CD44 and exhibited high efflux of Hoechst 33342 and Rhodamine 123 as a result of increased expression of ABCC1 (MRP1) transporters (76). It was also reported that Twist induced the activation of $\beta$-catenin signaling pathway and Akt pathways for the maintenance of the stem cell-like properties associated with EMT (77). Fang et al also found that Twist 2 not only promoted the EMT program, but also generated cells with stem cell-like properties (78). The ZEB1 transcription factor has been shown to modulate the two stemness genes KLF4 and SOX2 indirectly, via downregulation of
miR-200 which are rapidly emerging as master regulators of differentiation by directly targeting the transcriptional factors (ZEB1 and ZEB2/SIP1) to derepress E-cadherin and elicit mesenchymal-epithelial transition (MET), thus leads to the generation of migrating CSCs $(79,80)$. Guo et al revealed that Slug could cooperate with SOX9 in orchestrating the stem cell state (81). Collectively, these observations offer unquestionable evidence that EMT inducers are involved in regulating cancer cell stemness.

In addition, HER2-overexpressing breast carcinomas resistance to trastuzumab could also be linked to biology of stem cell-like cells. It was demonstrated that CD44 was overexpressed in trastuzumab resistant JIMT-1 cells and induced HER-2 receptor internalization in vitro and in vivo (82). Trastuzumab resistance can result from the spontaneous conversion of HER-2 ${ }^{+}$cells to a CD $44^{+} / \mathrm{CD} 24 \%$ HER-2 $\%$ low phenotype through EMT (83). It was discovered that trastuzumab sensitivity was restricted to the Slug/Snail2negative subset of luminal/HER $2^{+}$cell lines, whereas all of the Slug/Snail2-positive basal/HER $2^{+}$cell lines exhibited a primary (inherent) resistance to trastuzumab. Knockdown of Slug could suppress the CD $44^{+} / \mathrm{CD} 24 \%$ low phenotype which might be responsible for trastuzumab refractoriness in basal/ HER2 ${ }^{+}$JIMT1 cells and sensitize trastuzumab-resistant xenografts to trastuzumab. Quote of a sentence in the chapter: EMT-driving transcriptional repressor Slug/Snail2 appears to be a pivotal gene that induces an enhanced phenotypic plasticity in basal/HER2 ${ }^{+}$cells, thus allowing them to 'enter' into and 'exit' from trastuzumab-responsive stem cell-like states (84). Thus, EMT may promote drug-resistace via potentiating cell characteristics of CSCs.

\section{EMT, microRNAs and drug resistance in breast cancer}

MicroRNAs (miRNAs), a class of small cellular RNAs, acting as agents of the RNA interference pathway, can lead to silencing of their cognate target genes, by either cleaving mRNA molecules or inhibiting their translation (85). In this decade, studies have shown that miRNAs regulate EMT through directly targeting families of EMT transcription factors or affecting the integrity of the epithelial architecture during EMT progression (86). miR-200 family which has a striking negative correlation with ZEB could regulate EMT and drug resistance. It was reported that miR-200 expression could reverse resistance to EGFR inhibitor therapy in bladder cancer cells (87). miR-200 cluster was associated with substantial expression of E-cadherin mRNA in breast cancer tissues and low miR-200 expression was associated with pronounced benefits of cyclophosphamide (88). Restoration of miR-200c enhanced chemosensitivity to microtubule-directed agents in MCF-7 and T47D cells (89). In addition, miR-200c was shown to correlate with the acquired resistance of breast cancer cells to doxorubicin by inhibiting Akt signaling through its effects on E-cadherin and PTEN (90). A previous report found that transfection of MDA-MB-231 cells with pre-miR-200b or pre-miR-200c enhanced their sensitivity to doxorubicin. Similarly, reduced miRNA-200b and miR-200c expression contributed to endocrine resistance in breast cancer cells. Accompanied by the increase in miR-200b and miR-200c, ZEB1 expression was decreased and cells appeared more 
epithelial in morphology and were sensitized to TAM inhibition (91). It has been proved that miR-125b induced EMT-like molecular alterations, and functioned as a key mediator for Snail-induced stem cell propagation and chemoresistance in breast cancer cells $(92,93)$. A recent study reported EMT was linked to loss of ER $\alpha$ expression, through transcriptional repression of ER by Snail and concomitant translational inhibition of ER $\alpha$ mRNA by miR-221/222 (94). Additionally, miRNA-375 was found downregulated in the TAM-resistant MCF-7 cells, while its re-expression sensitized cells to TAM and reverted EMT-like properties. Metadherin (MTDH) was regarded as the direct target of miRNA-375, with its established relevance in drug resistance and breast cancer metastasis (95).

\section{Clinical prognostic of EMT and potential EMT-targeted therapy for breast cancer}

The transcriptional factors and the hallmarks of EMT are often related to more malignant type in breast cancer patients. High expression of Slug and Twist has been reported closely correlated with poor prognosis in patients with breast cancer $(96,97)$. Jeong et al noted that EMT was significantly related to high histological grade and triple-negative phenotype but not predictive of disease-free survival in patients with breast cancer (98). Since EMT has been established as a mechanism that confers tumor cells with the essential ability for drug resistance, metastasis, and acquired-tumor stem cell traits, inhibition of EMT can be a critical therapeutic strategy for prevention of tumor progression (99). NPI-0052, a protesome inhibitor, has been demonstrated to depress EMT via weakening NF- $\mathrm{BB}$ and Snail (100). Shinto et al found Ki26894, a TBR-I kinase inhibitor, suppressed the invasiveness and EMT in scirrhous gastric cancer cells (101). Artesunate (an antimalarial agent) has been discovered to induce cell cycle arrest and apoptosis possibly by affecting the hyperactive Wnt pathway and reversing EMT in colorectal cell lines (102). The Src kinase inhibitor dasatinib has been proven to inhibit growth of breast cancer cells with EMT features (103). Cystatin C, a cysteine protease inhibitor has been found to inhibit the acquisition of EMT and invasion stimulated by TGF- $\beta$ in breast cancer cell by preventing actin cytoskeletal rearrangements and E-cadherin downregulation (104). Chua et al developed an EMT inhibition screening assay to identify compounds targeting ALK5, MEK, and SRC as potent inhibitors that can interfere with EGF, HGF, or IGF-1 induced EMT signaling (105).

\section{Concluding remarks and future perspectives}

EMT is a complex, stepwise phenomenon that occurs during embryonic development and tumor progression, and involves major reprogramming of gene expression that leads to alterations in cell fate and behavior. Clarifying the underlying mechanism linking EMT and drug resistance would likely be useful for devising better targeted therapeutic approaches in combination with conventional therapeutics.

The hallmarks of EMT are loss of the epithelial molecule E-cadherin and gain of mesenchymal markers, such as $\mathrm{N}$-cadherin and vimentin. Loss of E-cadherin expression can lead to loss of contact inhibition, infinite proliferation, de-differentiation, loose intercellular connections, and be susceptibility to shedding in cancer cells, which enhances both invasion and migration of cancer cells (31). N-cadherin is highly expressed in invasive and metastatic human breast cancer cells and correlates with aggressive clinical behavior. The alterations of these genes contribute to endowing cells with higher malignancy. Moreover, an increasing number of direct evidence revealed these genes had close connection with the resistance to therapy (106-108). In addition, the transcriptional factors of EMT such as Snail, Slug and Twist not only elevate the cell invasion and metastasis to escape being killed, but also increase/decrease the essential genes taking part in drug resistance. Certain cytokines and genes play essential roles on both EMT and drug resistance. The signaling pathways of EMT are wide and extremely complex, which constitute main targets for novel drug development. A better understanding of the roles of EMT and CSCs in breast cancer will lead to more effective therapies that will target not only the tumor but also the residual population of cells that are responsible for the relapse and resurgence of the tumor. Further examination of the epigenetic changes such as miRNA will also be an important area of research. All these results suggest that drug combinations using conventional or targeted therapies together with targeting the EMT-related mechanisms need to be considered for winning the battle against drug resistant in cancer cells.

\section{Acknowledgments}

This study was supported by National Natural Science Foundation of China (No. 81102007 and No. 81472475), and the International S\&T Cooperation Program of China (ISTCP) (2012DFA10650).

\section{References}

1. Jemal A, Bray F, Center MM, Ferlay J, Ward E and Forman D: Global cancer statistics. CA Cancer J Clin 61: 69-90, 2011.

2. Hernandez-Aya LF and Gonzalez-Angulo AM: Adjuvant systemic therapies in breast cancer. Surg Clin North Am 93: 473-491, 2013.

3. Collignon J, Rorive A, Martin M, Andre C, Maweja S, Lifrange E, Coucke P and Jerusalem G: Systemic chemotherapy and breast cancer. Rev Med Liege 66: 372-378, 2011 (In French).

4. Saeki T, Tsuruo T, Sato W and Nishikawsa K: Drug resistance in chemotherapy for breast cancer. Cancer Chemother Pharmacol 56 (Suppl 1): 84-89, 2005

5. Coley HM: Mechanisms and strategies to overcome chemotherapy resistance in metastatic breast cancer. Cancer Treat Rev 34: 378-390, 2008.

6. Roxanis I: Occurrence and significance of epithelial-mesenchymal transition in breast cancer. J Clin Pathol 66: 517-521, 2013.

7. Wang Y and Zhou BP: Epithelial-mesenchymal transition - a hallmark of breast cancer metastasis. Cancer Hallm 1: 38-49, 2013.

8. Dave B, Mittal V, Tan NM and Chang JC: Epithelial-mesenchymal transition, cancer stem cells and treatment resistance. Breast Cancer Res 14: 202, 2012

9. Mallini P, Lennard T, Kirby J and Meeson A: Epithelial-tomesenchymal transition: What is the impact on breast cancer stem cells and drug resistance. Cancer Treat Rev 40: 341-348, 2014.

10. Szakács G, Paterson JK, Ludwig JA, Booth-Genthe C and Gottesman MM: Targeting multidrug resistance in cancer. Nat Rev Drug Discov 5: 219-234, 2006.

11. Gonzalez-Angulo AM, Morales-Vasquez F and Hortobagyi GN: Overview of resistance to systemic therapy in patients with breast cancer. Adv Exp Med Biol 608: 1-22, 2007.

12. Dean $\mathrm{M}: \mathrm{ABC}$ transporters, drug resistance, and cancer stem cells. J Mammary Gland Biol Neoplasia 14: 3-9, 2009. 
13. Shah PD and Dickler MN: Endocrine therapy for advanced breast cancer. Clin Adv Hematol Oncol 12: 214-223, 2014.

14. Normanno N, Di Maio M, De Maio E, De Luca A, de Matteis A, Giordano A and Perrone F; NCI-Naple Breast Cancer Group: Mechanisms of endocrine resistance and novel therapeutic strategies in breast cancer. Endocr Relat Cancer 12: 721-747, 2005.

15. Al Saleh S, Sharaf LH and Luqmani YA: Signalling pathways involved in endocrine resistance in breast cancer and associations with epithelial to mesenchymal transition (Review). Int J Oncol 38: 1197-1217, 2011.

16. Slamon D, Eiermann W, Robert N, Pienkowski T, Martin M, Press M, Mackey J, Glaspy J, Chan A, Pawlicki M, et al; Breast Cancer International Research Group: Adjuvant trastuzumab in HER2-positive breast cancer. N Engl J Med 365: 1273-1283, 2011.

17. Martin-Castillo B, Oliveras-Ferraros C, Vazquez-Martin A, Cufí S, Moreno JM, Corominas-Faja B, Urruticoechea A, Martín ÁG, López-Bonet E and Menendez JA: Basal/HER2 breast carcinomas: Integrating molecular taxonomy with cancer stem cell dynamics to predict primary resistance to trastuzumab (Herceptin). Cell Cycle 12: 225-245, 2013.

18. Gajria D and Chandarlapaty S: HER2-amplified breast cancer: Mechanisms of trastuzumab resistance and novel targeted therapies. Expert Rev Anticancer Ther 11: 263-275, 2011.

19. Foroni C, Broggini M, Generali D and Damia G: Epithelialmesenchymal transition and breast cancer: Role, molecular mechanisms and clinical impact. Cancer Treat Rev 38: 689-697, 2012.

20. Thiery JP and Sleeman JP: Complex networks orchestrate epithelial-mesenchymal transitions. Nat Rev Mol Cell Biol 7: 131-142, 2006

21. Voulgari A and Pintzas A: Epithelial-mesenchymal transition in cancer metastasis: Mechanisms, markers and strategies to overcome drug resistance in the clinic. Biochim Biophys Acta 1796: 75-90, 2009.

22. Bezdenezhnykh N, Semesiuk N, Lykhova O, Zhylchuk V and Kudryavets Y: Impact of stromal cell components of tumor microenvironment on epithelial-mesenchymal transition in breast cancer cells. Exp Oncol 36: 72-78, 2014.

23. Bonde AK, Tischler V,Kumar S, Soltermann A and SchwendenerRA Intratumoral macrophages contribute to epithelial-mesenchymal transition in solid tumors. BMC Cancer 12: 35, 2012.

24. Orimo A, Gupta PB, Sgroi DC, Arenzana-Seisdedos F, Delaunay T, Naeem R, Carey VJ, Richardson AL and Weinberg RA: Stroma fibroblasts present in invasive human breast carcinomas promote tumor growth and angiogenesis through elevated SDF-1/CXCL12 secretion. Cell 121: 335-348, 2005.

25. Yang J and Weinberg RA: Epithelial-mesenchymal transition: At the crossroads of development and tumor metastasis. Dev Cell 14: 818-829, 2008

26. Thiery JP, Acloque H, Huang RY and Nieto MA: Epithelialmesenchymal transitions in development and disease. Cell 139: 871-890, 2009

27. Vega S, Morales AV, Ocaña $\mathrm{OH}$, Valdés F, Fabregat I and Nieto MA: Snail blocks the cell cycle and confers resistance to cell death. Genes Dev 18: 1131-1143, 2004.

28. Kajita M, McClinic KN and Wade PA: Aberrant expression of the transcription factors snail and slug alters the response to genotoxic stress. Mol Cell Biol 24: 7559-7566, 2004.

29. Chen WJ, Wang H, Tang Y, Liu CL, Li HL and Li WT: Multidrug resistance in breast cancer cells during epithelial-mesenchyma transition is modulated by breast cancer resistant protein. Chin J Cancer 29: 151-157, 2010

30. Li W, Liu C, Tang Y, Li H, Zhou F and Lv S: Overexpression of Snail accelerates adriamycin induction of multidrug resistance in breast cancer cells. Asian Pac J Cancer Prev 12: 2575-2580, 2011.

31. Prasad CP, Rath G, Mathur S, Bhatnagar D, Parshad R and Ralhan R: Expression analysis of E-cadherin, Slug and GSK3beta in invasive ductal carcinoma of breast. BMC Cancer 9: 325, 2009.

32. Iseri OD, Kars MD, Arpaci F, Atalay C, Pak I and Gunduz U: Drug resistant MCF-7 cells exhibit epithelial-mesenchymal transition gene expression pattern. Biomed Pharmacother 65: 40-45, 2011.

33. Luqmani YA, Al Azmi A, Al Bader M, Abraham G and El Zawahri M: Modification of gene expression induced by siRNA targeting of estrogen receptor alpha in MCF7 human breast cancer cells. Int J Oncol 34: 231-242, 2009.

34. Andreeva OE, Shcherbakov AM, Shatskaia VA and Krasilńikov MA: The role of transcription factor Snaill in the regulation of hormonal sensitivity of in vitro cultured breast cancer cells. Vopr Onkol 58: 71-76, 2012 (In Russian).
35. Dhasarathy A, Kajita M and Wade PA: The transcription factor snail mediates epithelial to mesenchymal transitions by repression of estrogen receptor-alpha. Mol Endocrinol 21: 2907-2918, 2007.

36. Ye Y, Xiao Y, Wang W, Yearsley K, Gao JX and Barsky SH: ERalpha suppresses slug expression directly by transcriptional repression. Biochem J 416: 179-187, 2008.

37. Cheng GZ, Chan J, Wang Q, Zhang W, Sun CD and Wang LH: Twist transcriptionally up-regulates AKT2 in breast cancer cells leading to increased migration, invasion, and resistance to paclitaxel. Cancer Res 67: 1979-1987, 2007.

38. Li QQ, Xu JD, Wang WJ, Cao XX, Chen Q, Tang F, Chen ZQ, Liu XP and Xu ZD: Twist1-mediated adriamycin-induced epithelial-mesenchymal transition relates to multidrug resistance and invasive potential in breast cancer cells. Clin Cancer Res 15: 2657-2665, 2009.

39. Saxena M, Stephens MA, Pathak $H$ and Rangarajan A: Transcription factors that mediate epithelial-mesenchymal transition lead to multidrug resistance by upregulating $\mathrm{ABC}$ transporters. Cell Death Dis 2: e179, 2011

40. Bandyopadhyay A, Wang L, Agyin J, Tang Y, Lin S, Yeh IT, De K and Sun LZ: Doxorubicin in combination with a small TGFbeta inhibitor: A potential novel therapy for metastatic breast cancer in mouse models. PLoS One 5: e10365, 2010.

41. Hirohashi S and Kanai Y: Cell adhesion system and human cancer morphogenesis. Cancer Sci 94: 575-581, 2003.

42. López-Díaz FJ, Gascard P, Balakrishnan SK, Zhao J, Del Rincon SV, Spruck C, Tlsty TD and Emerson BM: Coordinate transcriptional and translational repression of p53 by TGF- $\beta 1$ impairs the stress response. Mol Cell 50: 552-564, 2013.

43. Shi XP, Miao S, Wu Y, Zhang W, Zhang XF, Ma HZ, Xin HL, Feng J, Wen AD and Li Y: Resveratrol sensitizes tamoxifen in antiestrogen-resistant breast cancer cells with epithelial-mesenchymal transition features. Int J Mol Sci 14: 15655-15668, 2013.

44. Wang SE, Xiang B, Guix M, Olivares MG, Parker J, Chung CH, Pandiella A and Arteaga CL: Transforming growth factor beta engages TACE and ErbB3 to activate phosphatidylinositol-3 kinase/Akt in ErbB2-overexpressing breast cancer and desensitizes cells to trastuzumab. Mol Cell Biol 28: 5605-5620, 2008.

45. Walsh LA and Damjanovski S: IGF-1 increases invasive potential of MCF-7 breast cancer cells and induces activation of latent TGF- $\beta 1$ resulting in epithelial to mesenchymal transition. Cell Commun Signal 9: 10, 2011.

46. Gooch JL, Van Den Berg CL and Yee D: Insulin-like growth factor (IGF)-I rescues breast cancer cells from chemotherapyinduced cell death - proliferative and anti-apoptotic effects. Breast Cancer Res Treat 56: 1-10, 1999.

47. Nahta R, Yuan LX, Zhang B, Kobayashi R and Esteva FJ: Insulinlike growth factor-I receptor/human epidermal growth factor receptor 2 heterodimerization contributes to trastuzumab resistance of breast cancer cells. Cancer Res 65: 11118-11128, 2005.

48. Gan Y, Wientjes MG and Au JL: Expression of basic fibroblast growth factor correlates with resistance to paclitaxel in human patient tumors. Pharm Res 23: 1324-1331, 2006.

49. McLeskey SW, Zhang L, El-Ashry D, Trock BJ, Lopez CA, Kharbanda S, Tobias CA, Lorant LA, Hannum RS, Dickson RB, et al: Tamoxifen-resistant fibroblast growth factor-transfected MCF-7 cells are cross-resistant in vivo to the antiestrogen ICI 182,780 and two aromatase inhibitors. Clin Cancer Res 4 697-711, 1998.

50. Magnani L, Stoeck A, Zhang X, Lánczky A, Mirabella AC, Wang TL, Gyorffy B and Lupien M: Genome-wide reprogramming of the chromatin landscape underlies endocrine therapy resistance in breast cancer. Proc Natl Acad Sci USA 110 E1490-E1499, 2013.

51. Zhang CC, Yan Z, Zong Q, Fang DD, Painter C, Zhang Q, Chen E, Lira ME, John-Baptiste A and Christensen JG: Synergistic effect of the $\gamma$-secretase inhibitor PF-03084014 and docetaxel in breast cancer models. Stem Cells Transl Med 2: 233-242, 2013.

52. Pandya K, Meeke K, Clementz AG, Rogowski A, Roberts J, Miele L, Albain KS and Osipo C: Targeting both Notch and ErbB-2 signalling pathways is required for prevention of ErbB-2-positive breast tumour recurrence. Br J Cancer 105: 796-806, 2011.

53. Orlowski RZ and Baldwin AS Jr: NF-kappaB as a therapeutic target in cancer. Trends Mol Med 8: 385-389, 2002.

54. Tapia MA, González-Navarrete I, Dalmases A, Bosch M, Rodriguez-Fanjul V, Rolfe M, Ross JS, Mezquita J, Mezquita C, Bachs $\mathrm{O}$, et al: Inhibition of the canonical IKK/NF kappa B pathway sensitizes human cancer cells to doxorubicin. Cell Cycle 6: 2284-2292,2007. 
55. Musgrove EA and Sutherland RL: Biological determinants of endocrine resistance in breast cancer. Nat Rev Cancer 9: 631-643, 2009.

56. Gee JM, Robertson JF, Ellis IO and Nicholson RI: Phosphorylation of ERK1/2 mitogen-activated protein kinase is associated with poor response to anti-hormonal therapy and decreased patient survival in clinical breast cancer. Int J Cancer 95: 247-254, 2001.

57. Berns K, Horlings HM, Hennessy BT, Madiredjo M, Hijmans EM, Beelen K, Linn SC, Gonzalez-Angulo AM, Stemke-Hale K, Hauptmann M, et al: A functional genetic approach identifies the PI3K pathway as a major determinant of trastuzumab resistance in breast cancer. Cancer Cell 12: 395-402, 2007.

58. Loh YN, Hedditch EL, Baker LA, Jary E, Ward RL and Ford CE: The Wnt signalling pathway is upregulated in an in vitro model of acquired tamoxifen resistant breast cancer. BMC Cancer 13: 174, 2013.

59. Wu Y, Ginther C, Kim J, Mosher N, Chung S, Slamon D and Vadgama JV: Expression of Wnt 3 activates Wnt/ $\beta$-catenin pathway and promotes EMT-like phenotype in trastuzumabresistant HER2-overexpressing breast cancer cells. Mol Cancer Res 10: 1597-1606, 2012.

60. Kumar A, Xu J, Brady S, Gao H, Yu D, Reuben J and Mehta K: Tissue transglutaminase promotes drug resistance and invasion by inducing mesenchymal transition in mammary epithelial cells. PLoS One 5: e13390, 2010.

61. Liu Y, Du F, Chen W, Yao M, Lv K and Fu P: Knockdown of dual specificity phosphatase 4 enhances the chemosensitivity of MCF-7 and MCF-7/ADR breast cancer cells to doxorubicin. Exp Cell Res 319: 3140-3149, 2013

62. Kim MR, Choi HK, Cho KB, Kim HS and Kang KW: Involvement of Pin1 induction in epithelial-mesenchymal transition of tamoxifen-resistant breast cancer cells. Cancer Sci 100: 1834-1841, 2009

63. Asiedu MK, Beauchamp-Perez FD, Ingle JN, Behrens MD, Radisky DC and Knutson KL: AXL induces epithelial-tomesenchymal transition and regulates the function of breast cancer stem cells. Oncogene 33: 1316-1324, 2014.

64. Dontu G, Abdallah WM, Foley JM, Jackson KW, Clarke MF, Kawamura MJ and Wicha MS: In vitro propagation and transcriptional profiling of human mammary stem/progenitor cells. Genes Dev 17: 1253-1270, 2003.

65. Donnenberg VS and Donnenberg AD: Multiple drug resistance in cancer revisited: The cancer stem cell hypothesis. J Clin Pharmacol 45: 872-877, 2005.

66. Jordan CT, Guzman ML and Noble M: Cancer stem cells. N Engl J Med 355: 1253-1261, 2006

67. Kakarala M and Wicha MS: Implications of the cancer stem-cell hypothesis for breast cancer prevention and therapy. J Clin Oncol 26: 2813-2820, 2008

68. Morel AP, Lièvre M, Thomas $\mathrm{C}$, Hinkal G, Ansieau S and Puisieux A: Generation of breast cancer stem cells through epithelial-mesenchymal transition. PLoS One 3: e2888, 2008

69. Radisky DC and LaBarge MA: Epithelial-mesenchymal transition and the stem cell phenotype. Cell Stem Cell 2: 511-512, 2008.

70. Santisteban M, Reiman JM, Asiedu MK, Behrens MD, Nassar A, Kalli KR, Haluska P, Ingle JN, Hartmann LC, Manjili MH, et al: Immune-induced epithelial to mesenchymal transition in vivo generates breast cancer stem cells. Cancer Res 69: 2887-2895, 2009.

71. Aktas B, Tewes M, Fehm T, Hauch S, Kimmig R and KasimirBauer S: Stem cell and epithelial-mesenchymal transition markers are frequently overexpressed in circulating tumor cells of metastatic breast cancer patients. Breast Cancer Res 11: R46, 2009.

72. Lee JM, Dedhar S, Kalluri R and Thompson EW: The epithelialmesenchymal transition: New insights in signaling, development, and disease. J Cell Biol 172: 973-981, 2006.

73. Taube JH, Herschkowitz JI, Komurov K, Zhou AY, Gupta S, Yang J, Hartwell K, Onder TT, Gupta PB, Evans KW, et al: Core epithelial-to-mesenchymal transition interactome gene-expression signature is associated with claudin-low and metaplastic breast cancer subtypes. Proc Natl Acad Sci USA 107: 15449-15454, 2010.

74. Mani SA, Guo W, Liao MJ, Eaton EN, Ayyanan A, Zhou AY, Brooks M, Reinhard F, Zhang CC, Shipitsin M, et al: The epithelial-mesenchymal transition generates cells with properties of stem cells. Cell 133: 704-715, 2008.

75. Wielenga VJ, Smits R, Korinek V, Smit L, Kielman M, Fodde R, Clevers H and Pals ST: Expression of CD44 in Apc and Tcf mutant mice implies regulation by the WNT pathway. Am J Pathol 154: 515-523, 1999.
76. Vesuna F, Lisok A, Kimble B and Raman V: Twist modulates breast cancer stem cells by transcriptional regulation of CD24 expression. Neoplasia 11: 1318-1328, 2009.

77. Li J and Zhou BP: Activation of $\beta$-catenin and Akt pathways by Twist are critical for the maintenance of EMT associated cancer stem cell-like characters. BMC Cancer 11: 49, 2011.

78. Fang X, Cai Y, Liu J, Wang Z, Wu Q, Zhang Z, Yang CJ, Yuan L and Ouyang G: Twist 2 contributes to breast cancer progression by promoting an epithelial-mesenchymal transition and cancer stem-like cell self-renewal. Oncogene 30: 4707-4720, 2011.

79. Wellner U, Schubert J, Burk UC, Schmalhofer O, Zhu F, Sonntag A, Waldvogel B, Vannier C, Darling D, zur Hausen A, et al: The EMT-activator ZEB1 promotes tumorigenicity by repressing stemness-inhibiting microRNAs. Nat Cell Biol 11: $1487-1495,2009$

80. Park SM, Gaur AB, Lengyel E and Peter ME: The miR-200 family determines the epithelial phenotype of cancer cells by targeting the E-cadherin repressors ZEB1 and ZEB2. Genes Dev 22: 894-907, 2008

81. Guo W, Keckesova Z, Donaher JL, Shibue T, Tischler V, Reinhardt F, Itzkovitz S, Noske A, Zürrer-Härdi U, Bell G, et al: Slug and Sox 9 cooperatively determine the mammary stem cell state. Cell 148: 1015-1028, 2012.

82. Pályi-Krekk Z, Barok M, Isola J, Tammi M, Szöllosi J and Nagy P: Hyaluronan-induced masking of ErbB2 and CD44enhanced trastuzumab internalisation in trastuzumab resistant breast cancer. Eur J Cancer 43: 2423-2433, 2007.

83. Lesniak D, Sabri S, Xu Y, Graham K, Bhatnagar P, Suresh M and Abdulkarim B: Spontaneous epithelial-mesenchymal transition and resistance to HER-2-targeted therapies in HER-2-positive luminal breast cancer. PLoS One 8: e71987, 2013.

84. Oliveras-Ferraros C, Corominas-Faja B, Cufí S, VazquezMartin A, Martin-Castillo B, Iglesias JM, López-Bonet E, Martin ÁG and Menendez JA: Epithelial-to-mesenchymal transition (EMT) confers primary resistance to trastuzumab (Herceptin). Cell Cycle 11: 4020-4032, 2012

85. Bartel DP: MicroRNAs: Genomics, biogenesis, mechanism, and function. Cell 116: 281-297, 2004.

86. Lamouille S, Subramanyam D, Blelloch R and Derynck R: Regulation of epithelial-mesenchymal and mesenchymal-epithelial transitions by microRNAs. Curr Opin Cell Biol 25: 200-207, 2013.

87. Adam L, Zhong M, Choi W, Qi W, Nicoloso M, Arora A, Calin G, Wang H, Siefker-Radtke A, McConkey D, et al: miR-200 expression regulates epithelial-to-mesenchymal transition in bladder cancer cells and reverses resistance to epidermal growth factor receptor therapy. Clin Cancer Res 15: 5060-5072, 2009.

88. Bojmar L, Karlsson E, Ellegård S, Olsson H, Björnsson B, Hallböök O, Larsson M, Stål O and Sandström P: The role of microRNA-200 in progression of human colorectal and breast cancer. PLoS One 8: e84815, 2013.

89. Cochrane DR, Spoelstra NS, Howe EN, Nordeen SK and Richer JK: MicroRNA-200c mitigates invasiveness and restores sensitivity to microtubule-targeting chemotherapeutic agents. Mol Cancer Ther 8: 1055-1066, 2009.

90. Chen Y, Sun Y, Chen L, Xu X, Zhang X, Wang B, Min L and Liu W: miRNA-200c increases the sensitivity of breast cancer cells to doxorubicin through the suppression of E-cadherinmediated PTEN/Akt signaling. Mol Med Rep 7: 1579-1584, 2013.

91. Manavalan TT, Teng Y, Litchfield LM, Muluhngwi P, Al-Rayyan N and Klinge CM: Reduced expression of miR-200 family members contributes to antiestrogen resistance in LY2 human breast cancer cells. PLoS One 8: e62334, 2013.

92. Liu Z, Liu H, Desai S, Schmitt DC, Zhou M, Khong HT, Klos KS, McClellan S, Fodstad O and Tan M: miR-125b functions as a key mediator for snail-induced stem cell propagation and chemoresistance. J Biol Chem 288: 4334-4345, 2013.

93. Wang HJ, Guo YQ, Tan G, Dong L, Cheng L, Li KJ, Wang ZY and Luo HF: miR-125b regulates side population in breast cancer and confers a chemoresistant phenotype. J Cell Biochem 114: 2248-2257, 2013.

94. Guttilla IK, Phoenix KN, Hong X, Tirnauer JS, Claffey KP and White BA: Prolonged mammosphere culture of MCF-7 cells induces an EMT and repression of the estrogen receptor by microRNAs. Breast Cancer Res Treat 132: 75-85, 2012.

95. Ward A, Balwierz A, Zhang JD, Küblbeck M, Pawitan Y, Hielscher T, Wiemann S and Sahin Ö: Re-expression of microRNA-375 reverses both tamoxifen resistance and accompanying EMT-like properties in breast cancer. Oncogene 32: $1173-1182,2013$ 
96.Liu T, Zhang X, Shang M, Zhang Y, Xia B, Niu M, Liu Y and Pang D: Dysregulated expression of Slug, vimentin, and E-cadherin correlates with poor clinical outcome in patients with basal-like breast cancer. J Surg Oncol 107: 188-194, 2013.

97. Soini Y, Tuhkanen H, Sironen R, Virtanen I, Kataja V, Auvinen P, Mannermaa A and Kosma VM: Transcription factors zeb1, twist and snail in breast carcinoma. BMC Cancer 11: 73, 2011.

98. Jeong H, Ryu YJ, An J, Lee Y and Kim A: Epithelialmesenchymal transition in breast cancer correlates with high histological grade and triple-negative phenotype. Histopathology 60B: E87-E95, 2012.

99. Thiery JP: Epithelial-mesenchymal transitions in tumour progression. Nat Rev Cancer 2: 442-454, 2002.

100. Baritaki S, Chapman A, Yeung K, Spandidos DA, Palladino M and Bonavida B: Inhibition of epithelial to mesenchymal transition in metastatic prostate cancer cells by the novel proteasome inhibitor, NPI-0052: Pivotal roles of Snail repression and RKIP induction. Oncogene 28: 3573-3585, 2009.

101. Shinto O, Yashiro M, Kawajiri H, Shimizu K, Shimizu T, Miwa A and Hirakawa K: Inhibitory effect of a TGFbeta receptor type-I inhibitor, Ki26894, on invasiveness of scirrhous gastric cancer cells. Br J Cancer 102: 844-851, 2010.

102. Li L-N, Zhang H-D, Yuan S-J, Yang D-X, Wang L and Sun Z-X: Differential sensitivity of colorectal cancer cell lines to artesunate is associated with expression of beta-catenin and E-cadherin. Eur J Pharmacol 588: 1-8, 2008.
103. Sokol JP, Neil JR, Schiemann BJ and Schiemann WP: The use of cystatin $C$ to inhibit epithelial-mesenchymal transition and morphological transformation stimulated by transforming growth factor-beta. Breast Cancer Res 7: R844-R853, 2005.

104. Finn RS, Dering J, Ginther C, Wilson CA, Glaspy P, Tchekmedyian N and Slamon DJ: Dasatinib, an orally active small molecule inhibitor of both the src and abl kinases, selectively inhibits growth of basal-type/'triple-negative' breast cancer cell lines growing in vitro. Breast Cancer Res Treat 105: 319-326, 2007.

105. Chua KN, Sim WJ, Racine V, Lee SY, Goh BC and Thiery JP: A cell-based small molecule screening method for identifying inhibitors of epithelial-mesenchymal transition in carcinoma. PLoS One 7: e33183, 2012.

106. Lu L, Zhou D, Jiang X, Song K, Li K and Ding W: Loss of E-cadherin in multidrug resistant breast cancer cell line MCF-7/ Adr: Possible implication in the enhanced invasive ability. Eur Rev Med Pharmacol Sci 16: 1271-1279, 2012.

107. Roger L, Jullien L, Gire V and Roux P: Gain of oncogenic function of p53 mutants regulates E-cadherin expression uncoupled from cell invasion in colon cancer cells. J Cell Sci 123: 1295-1305, 2010.

108. Zhang B, Groffen J and Heisterkamp N: Increased resistance to a farnesyltransferase inhibitor by $\mathrm{N}$-cadherin expression in Bcr/Abl-P190 lymphoblastic leukemia cells. Leukemia 21: 1189-1197, 2007. 